\title{
Local structural excitations in model glasses
}

\author{
S. Swayamjyoti, ${ }^{1}$ J. F. Löffler, ${ }^{2}$ and P. M. Derlet ${ }^{1, *}$ \\ ${ }^{1}$ Condensed Matter Theory Group, Paul Scherrer Institut, 5232 Villigen PSI, Switzerland \\ ${ }^{2}$ Laboratory of Metal Physics and Technology, Department of Materials, ETH Zurich, 8093 Zurich, Switzerland
}

(Received 14 February 2014; revised manuscript received 20 May 2014; published 13 June 2014)

\begin{abstract}
Structural excitations of model Lennard-Jones glass systems are investigated using the activation-relaxation technique (ARTn), which explores the potential energy landscape around a local minimum energy configuration by converging to a nearby saddle-point configuration. Performing ART $n$ results in a distribution of barrier energies that is single-peaked for well-relaxed samples. The present work characterizes such atomic-scale excitations in terms of their local structure and environment. It is found that, at zero applied stress, many of the identified events consist of chainlike excitations that can either be extended or ringlike in their geometry. The location and barrier energy of these saddle-point structures are found to correlate with the type of atom involved, and with spatial regions that have low Kelvin eigenshear moduli and are close to the excess free volume within the configuration. Such correlations are, however, weak and more generally the identified local structural excitations are seen to exist throughout the model glass sample. The work concludes with a discussion within the framework of $\alpha$ and $\beta$ relaxation processes that are known to occur in the undercooled liquid regime.
\end{abstract}

DOI: 10.1103/PhysRevB.89.224201

PACS number(s): 61.43.Fs, 83.80.Ab, 62.20.F-

\section{INTRODUCTION}

Local structural excitations (LSEs) occurring at the atomic scale of bulk metallic glasses (BMGs) are believed to mediate their unique mechanical properties. In the early work of Spaepen [1], a free-volume model was proposed in which single atoms would migrate to a neighboring free-volume region. Subsequently, Argon [2] introduced the idea that small groups of atoms undergo a structural transformation that could modify the local shear-stress state. The early atomistic simulations of Falk and Langer [3] confirmed this picture and referred to these LSEs as shear transformation zones (STZs), leading to a series of static and dynamical atomistic simulations to better understand how such STZs lead collectively to macroscopic plasticity [4-8]. The structural transformations studied in these works were obtained via application of strain rates that are many orders of magnitude higher than that normally seen in experiment, leading to localized material instabilities often identified as STZs. In terms of the traditional thermal activation picture for plasticity, such simulations therefore study the athermal limit of plasticity in bulk metallic glasses.

A common question has been as to whether there exist local structural features within the glass configuration that facilitate the existence of LSEs. Indeed, from the early athermal atomistic simulation work, those spatial regions that generated a localized material instability and the subsequent observation of STZs have been referred to as pre-existing liquidlike regions-spatial domains that are not fully relaxed [9-12]. In the work of Langer, a relatively low density of such regions was theoretically considered [12]. From this perspective, it is then valid to ask whether there are structural features of the unstrained glass configuration that allow identification of regions predisposed to LSE's-the so-called liquidlike regions? In the work of Léonforte et al. [13], reversible nonaffine atomic displacements associated with a finite elastic distortion were

\footnotetext{
*Corresponding author: Peter.derlet@psi.ch
}

considered. Such displacements give insight into the spatial extent of the low curvature of the unstrained potential energy landscape (PEL). This work found a percolative network of large nonaffine displacements. However, when the applied strain was increased to promote plastic activity, the location of the observed structural changes did not show a strong correlation with the nonaffine displacements, suggesting that the corresponding athermal energy barriers were not directly related to the unstrained PEL curvature. Mayr [14], who analyzed the local elastic constants (Born plus the kinetic fluctuation contributions) as a function of applied stress and temperature, found a strong connection between emerging elastic instabilities and eventual plasticity as the temperature approached the glass transition. While these simulations were fully dynamical, the necessarily high strain rates place these results close to the athermal regime.

Atomistic simulation methods that avoid the high-stress athermal limit are the so-called PEL exploration methods. The relevance of these methods to BMGs relies on the idea that the atomic configuration of a structural glass spends much of its time in a local potential energy minimum, only occasionally transiting it via a saddle-point region to a new local minimum. Such structural changes are assumed to occur via thermal fluctuations and therefore do not involve a local material instability driven by the applied stress. A well-known example of this approach is the activation-relaxation technique nouveau (ARTn) [15-17], which has been applied to both two- and three-dimensional model glass systems [18-21]. Starting from a well-defined local minimum, the ART $n$ method uses the local Hessian structure of the PEL to climb out of its current PEL valley and to eventually converge to a nearby saddle point region. The energy difference between the minimum and the saddle point gives the corresponding energy barrier of the associated LSE. When applied to model glass systems a wide range of energy barriers are obtained producing a distribution of energy barriers that appear to converge when several thousand of such LSEs have been identified. For sufficiently relaxed glass configurations, the barrier energy distribution is found to be single-peaked and 
analysis of the associated LSEs revealed their corresponding plastic strain increments to be uncorrelated with barrier energy [18]. Moreover, with respect to a particular loading geometry the plastic strains have equal numbers of positive and negative sign. However, upon application of the load, the distribution becomes slightly weighted towards positive strains with a large number of negative strain LSEs still occurring. The situation is quite different for the case of stress-driven energy barriers, which generally only yield LSEs that are positive in strain. Thus athermal stress-driven simulations probe only a subset of possible LSEs - those compatible with the driving mode chosen.

In this paper, the ARTn method is applied to threedimensional model Lennard Jones (LJ) structural glass configurations to obtain a large number of LSEs and their associated barrier energies. The present work will only consider the case of zero external load. Using these data, the spatial location of the identified LSEs will be investigated in terms of local structural properties and a statistical analysis will be performed. The local quantities investigated will be atom type, local energy, local elastic constants, local pressure, and Voronoi volume. In addition, the spatial nature of the structural changes and the number of atoms involved will be determined. All such information will also be correlated with the barrier energy. Additionally, the correlation between the spatial extent of the LSEs and that of the low-frequency vibrational modes will be investigated, since the latter is known to correlate with elastically soft regions [22]. In Sec. II the sample preparation procedure will be outlined and the local structural quantities to be investigated defined. Section III contains the major results of the present work encompassing the ART $n$ results and the ensuing statistical analysis of the identified LSEs. In Sec. IV, the spatial nature of a number of representative LSEs will be atomistically visualized and characterized. Finally, Sec. V will discuss the results in terms of contemporary pictures of microscopic plastic deformation in BMGs.

\section{METHODOLOGY}

\section{A. Sample preparation and ART $n$ calculations}

Model glass samples have been prepared by molecular dynamics and statics using a 50/50 binary mixture of a $\mathrm{LJ}$ system, which is defined by the interatomic potential,

$$
V_{\mathrm{LJ}}(r)=4 \epsilon\left[\left(\frac{\sigma_{\alpha \beta}}{r}\right)^{12}-\left(\frac{\sigma_{\alpha \beta}}{r}\right)^{6}\right],
$$

where $\epsilon$ and $\sigma$ set the microscopic energy and length scale of the model material. The parametrization presently used is that of Wahnström parametrization [23]. All simulation results are reported in LJ units where time is measured with respect to $\tau=\sqrt{m \sigma^{2} / \varepsilon}$ and temperature with respect to $\varepsilon / k_{\mathrm{b}}$.

Four samples, each with 1728 atoms, are prepared using different quench rates $\left(\eta_{1}=24.57 / 500, \eta_{2}=24.57 / 5000\right.$, $\eta_{3}=24.57 / 50000$, and $\left.\eta_{4}=24.57 / 50000\right)$. The sample preparation involves three steps: (1) equilibration of the liquid state by constant particle number, pressure, and temperature (NPT) molecular dynamics at a temperature of $10000 \times k_{b}$ $\left[\varepsilon / k_{b}\right]$ and hydrostatic pressure of $8 / 160\left[\varepsilon / \sigma_{11}^{3}\right]$, (2) slow quenching of the sample from this well-equilibrated liquid state, which involves an incremental reduction in both temperature $\left(-198.0 \times k_{b}\left[\varepsilon / k_{b}\right]\right)$ and pressure $\left(-0.158 / 160\left[\varepsilon / \sigma_{11}^{3}\right]\right)$ by NPT molecular dynamics to form the disordered amorphous glass at a temperature of $100 \times k_{b}\left[\varepsilon / k_{b}\right]$ and hydrostatic pressure of $0.1 / 160\left[\varepsilon / \sigma_{11}^{3}\right]$, and (3) relaxation of the atomic coordinates to zero temperature and zero hydrostatic pressure by molecular statics using the Parrinello-Rahman method [24]. For steps 1 and 2, the Parrinello-Rahman [24] barostat is used for pressure control and the Anderson-Hoover [25] thermostat is used for temperature control.

The ART $n$ technique is then applied to these samples. The ART $n$ identification of an LSE involves randomly choosing one atom and displacing it by a small distance $(<0.1 \sigma)$ from its equilibrium position-the starting condition for ART $n$. The lowest eigenvalue of the corresponding Hessian matrix is determined and the system is moved along the corresponding $3 \mathrm{~N}$ dimensional eigenvector until the eigenvalue of the Hessian becomes negative. This part of the algorithm is referred to as the "activation" phase. At this point, the configuration has passed an inflection region of the PEL and enters the new phase of "relaxation." Here, the configuration is moved in the direction of the eigenvector of the lowest eigenvalue, which is now negative, with a new eigenvalue and eigenvector to be calculated at each iteration. This is repeated until the dot product of the total force of the configuration with the eigenvector is zero. When this occurs, the configuration has reached a saddle-point which, following Ref. [18], is referred to as the activated state. It should be emphasized here that it is assumed that ART $n$ provides an unbiased probe to nearby saddle-point configurations within the PEL-an assumption that underlies all previous work applying ART $n$ to structural glasses [18-21].

To study the atomic scale environment of each identified LSE, an appropriate atomic weight for the $i$ th atom is calculated via the displacement vectors (referred to as $\Delta \mathbf{R}_{i}$ ) between the initial state and either the activated or the final state configuration. Presently, the normalised weights (which sum to unity) are defined as

$$
w_{i}=\frac{\left|\Delta \mathbf{R}_{i}\right|^{4}}{\sum_{i=1}^{N}\left|\Delta \mathbf{R}_{i}\right|^{4}} .
$$

This form is motivated by the standard definition of the participation number (PN) of an eigenvector [26], which gives information about the number of elements contributing to the norm of the vector. Indeed,

$$
\mathrm{PN}=\frac{1}{\sum_{i=1}^{N} w_{i}^{2}}
$$

gives the effective number of atoms involved in the identified LSE.

Given a local atomic quantity (LAQ) for each atom, the weighted average of the quantity, according to Eq. (2), gives a representative value for the region occupied by a particular LSE. That is,

$$
\mathrm{LAQ}_{\mathrm{LSE}}=\sum_{i=1}^{N} w_{i} \times \mathrm{LAQ}_{i} .
$$

With this quantity, an average over many LSEs can be made and compared to the unweighted LAQ average where all 
atoms equally contribute to the average. Scatter diagrams for the weighted LAQ with respect to barrier energy are also investigated to determine if any correlation exists between local structural features and the barrier energy of an LSE. To find out any possible linear correlation of such plots, the Pearson coefficient (PC) is used. For $n$ data points, this is given by

$$
\mathrm{PC}=\frac{\sum_{i=1}^{n}\left(X_{i}-\bar{X}\right)\left(Y_{i}-\bar{Y}\right)}{\sqrt{\sum_{i=1}^{n}\left(X_{i}-\bar{X}\right)^{2}} \sqrt{\sum_{i=1}^{n}\left(Y_{i}-\bar{Y}\right)^{2}}},
$$

where $X_{i}$ and $Y_{i}$ are the data sets, and $\bar{X}$ and $\bar{Y}$ are their respective arithmetic means. The value of $\mathrm{PC}$ ranges from -1 to 1 , where the extremal values -1 and 1 refer to a perfect linear correlation, and a value of zero indicates no linear correlation.

\section{B. Local atomic quantities}

The local atomic quantities (the LAQs) presently considered are (1) volume, calculated via an atomic scale Voronoi tessellation using the voro++ package [27], (2) energy, (3) pressure, (4) dilation elastic modulus (three times the local bulk modulus), and (5) the five linearly independent Kelvin eigenshear elastic moduli.

Since a LJ pair potential is presently being used, the expressions for the local stress and elastic moduli are of a simple form, where

$$
\sigma_{a}^{\mu \nu}=\frac{1}{2 V_{a}} \sum_{i j} V^{\prime}\left(R_{i j}\right) \frac{R_{i j}^{\mu} R_{i j}^{v}}{R_{i j}} \Lambda_{a, i j}
$$

and

$$
\begin{aligned}
C_{a}^{\mu \nu \alpha \beta}= & \frac{1}{2 V_{a}} \sum_{i j}\left[V^{\prime \prime}\left(R_{i j}\right)-\frac{V^{\prime}\left(R_{i j}\right)}{R_{i j}}\right] \frac{R_{i j}^{\mu} R_{i j}^{\nu} R_{i j}^{\alpha} R_{i j}^{\beta}}{R_{i j}^{2}} \Lambda_{a, i j} \\
& +\sigma_{a}^{\nu \beta} \delta_{\mu \alpha}+\sigma_{a}^{\nu \alpha} \delta_{\mu \beta} .
\end{aligned}
$$

In the above expressions, $\Lambda_{a, i j}$ represents the proportion of the $i j$ th bond within the volume of atom $a$. It is noted that bonds between two atoms, neither of which is atom $a$, may also contribute to these two local quantities. Equations (6) and (7) properly partition volume and therefore correctly take into account the contribution of each atomic bond [28,29]. The local pressure is obtained by taking one-third the trace of the local shear stress tensor. To obtain the Kelvin elastic moduli [30] from the fourth rank elastic stiffness tensor [Eq. (7)], the usual Voigt elastic stiffness matrix is first constructed from which the Kelvin matrix (second-rank tensor) is obtained via $C_{K}^{\mu \nu}=A^{\mu \nu} C_{V}^{\mu \nu}$. For an explicit form of $A^{\mu \nu}$, see Ref. [22]. The five linearly independent eigenshear moduli are obtained by first projecting out the pure dilation distortions and then diagonalizing the resulting Kelvin matrix-for more details, see Refs. [14,22].

In contrast to the popular Voigt notation, the Kelvin notation preserves the norm of the actual elastic stiffness tensor and hence their eigenvalues and eigenvectors have geometrical significance [31]. Since the Kelvin matrix is a tensor, the eigenvalues of the stiffness matrix can be computed to obtain the bulk modulus and the five linearly independent eigenshear moduli. The invariance of these eigenshear moduli with respect to coordinate systems and thereby their role as an intrinsic material property has been highlighted in Ref. [32].

\section{Natural mode analysis}

The natural modes of an $N$ atom configuration can be obtained via the solution to

$$
\sum_{j v}\left(m_{i}\left[\omega_{n}\right]^{2} \delta_{i j} \delta^{\mu v}-\Delta_{i j}^{\mu \nu}\right) u_{j, n}^{v}=0,
$$

where $\Delta_{i j}^{\mu \nu}$ is the translationally invariant dynamical matrix obtained from

$$
\Delta_{i j}^{\mu \nu}=\sum_{a, a \neq i} H_{i a}^{\mu v} \delta_{i j}-H_{i j}^{\mu \nu}\left(1-\delta_{i j}\right) .
$$

Here, $H_{i j}^{\mu \nu}$ is the Hessian. In terms of the LJ interaction $V(r)$, the Hessian may be written as

$$
H_{i j}^{\mu \nu}=\left[V^{\prime \prime}\left(R_{i j}\right)-\frac{V^{\prime}\left(R_{i j}\right)}{R_{i j}}\right] \frac{R_{i j}^{\mu} R_{i j}^{\nu}}{R_{i j}^{2}}+\frac{V^{\prime}\left(R_{i j}\right)}{R_{i j}} \delta^{\mu \nu} .
$$

In Eq. (8), $m_{i}$ is the atomic mass of the $i$ th atom, and $u_{j, n}^{v}$ is the eigenvector associated with the eigenfrequency $\omega_{n}$ of the $n$th natural mode.

The vibrational density of states (VDOS) may be formally defined via

$$
\rho_{\mathrm{VDOS}}(\omega)=\sum_{n} \delta\left(\omega-\omega_{n}\right)
$$

where $\delta(\cdots)$ is the Dirac $\delta$ function which, in practice, is replaced by a continuous function of finite width. The number of atoms participating in a particular eigenstate, $u_{j, n}^{v}$, may be obtained via the vibrational participation number [26]

$$
\mathrm{PN}_{n}=\left(\sum_{i}\left|\vec{u}_{i, n}\right|^{4}\right)^{-1}
$$

where $\vec{u}_{i, n}$ is the three-dimensional polarization vector of atom $i$ coming from the eigenvector of eigenfrequency $\omega_{n}$. Assuming a normalized eigenvector, $\mathrm{PN}_{n}$ will range between unity (when the eigenstate is concentrated on just one atom) and $N$ (when the eigenstate is distributed evenly over the entire sample).

\section{RESULTS}

\section{A. ARTn}

In total 4262 unique activated states are identified using the ART $n$ method. To verify that each activated state is directly connected to the initial atomic configuration, the activated configuration is perturbed in a direction towards the initial atomic state configuration and allowed to relax. If the resulting structure differed from the initial structure the LSE is discarded from the data set, as was done in Ref. [18]. In a similar way, the final state is determined by perturbing the activated configuration in a direction away from the initial atomic state configuration and allowed to relax.

To determine the spatial location of a particular LSE, the center-of-position of those saddle-point atoms displaced relative to the initial configuration by more than $0.1 \sigma$ is calculated. Figure 1 displays these positions within a boundary 


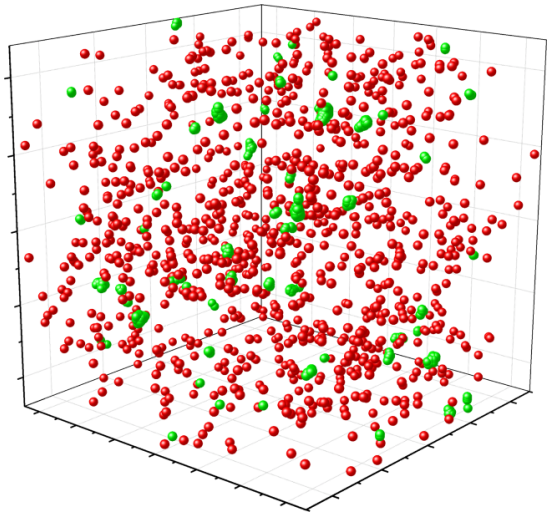

FIG. 1. (Color online) Red balls represent the center of positions of all identified LSEs and the green balls represent regions containing free-volume within the simulation cell of the model glass.

box defining the three-dimensional periodic simulation cell. A general inspection of their spatial distribution reveals some heterogeneity and, in particular, regions where many LSEs are located in very close proximity. A more detailed inspection of such LSEs (as in a manner described in Sec. IV) reveals them to be quite different in spatial extent and barrier energy, despite some LSEs having their center of position almost coincident. The goal of the proceeding section will be to see if any local structural feature correlates with this observed heterogeneity.

Figure 2(a) displays the distribution of barrier energies obtained from the 4262 identified activated states for the sample with the slowest quench rate. In agreement with Refs. [18-21], the distribution peaks at a nonzero barrier energy and appears to approach zero for small enough barrier energies. The barrier energy scale is comparable to that seen in a previous fully three-dimensional ART $n$ simulation using the same LJ potential parametrization [21].

Figure 2(b) shows a histogram of the LSE participation number, indicating that the LSEs identified by ART $n$ generally involve one to several atoms. Histograms are shown for both the activated and final state configurations demonstrating that little difference occurs in the statistics of the number of atoms involved when considering either the activated or the final state configurations. Inspection of a given saddle point and its corresponding final state PN did show either an increase
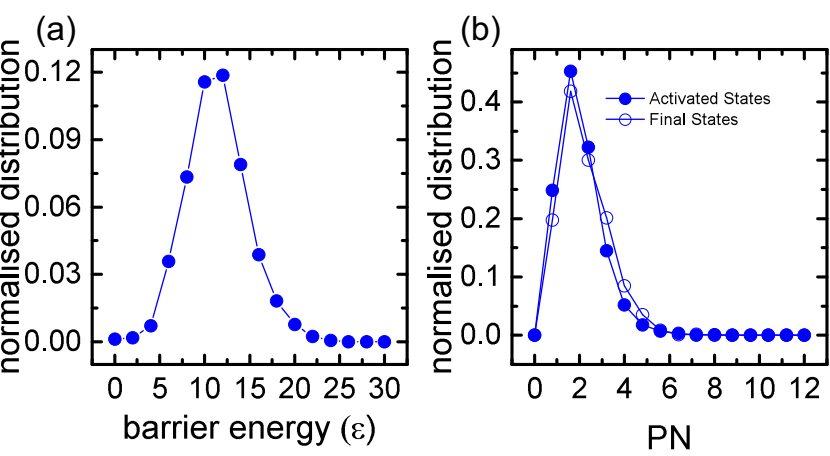

FIG. 2. (Color online) (a) Normalized distribution of barrier energies of LSEs in a 3D model glass system. (b) Histogram of participation number. or decrease of participating atoms. However, on average, the change in the PN is close to zero indicating no strong bias to whether the final state contains more or less participating atoms than the activated state. A scatter diagram (not shown) of PN and barrier energy revealed no strong correlation with barrier energy. It is noted that detailed inspection of such a scatter plot revealed final state PNs which exactly equaled an integer. Subsequent visual inspection of these LSEs revealed them to involve a permutation of nearby atoms of the same type, where the activated configuration involved a closed loop of displaced neighboring atoms [see Fig. 7(a), for an example]. Because such LSEs (which numbered 389) cannot produce any strain these are removed from the data set used in the LAQ analysis of the proceeding section.

\section{B. Statistical analysis of LAQs}

Figure 3 displays the normalized histograms of a number of LSE-averaged LAQs with respect to the activated and final state configurations. Also shown are the equivalent unweighted histograms derived from the total sample. The two types of distributions will be referred to as LSE-weighted and unweighted distributions of the LAQ. For the unweighted distributions, the (atom-type resolved) partial histograms are also shown.

Figure 3(a) represents the normalized distribution of potential energy of atoms. The double peak structure of the total unweighted distribution is clearly seen to arise from the single-peaked distributions of each atomic type. The LSE-weighted distributions, on the other hand, do not exhibit a double-peak structure, with the single observed peak coinciding with the unweighted partial distribution of atoms of type 2. This result suggests that the atoms involved in an LSE (a)
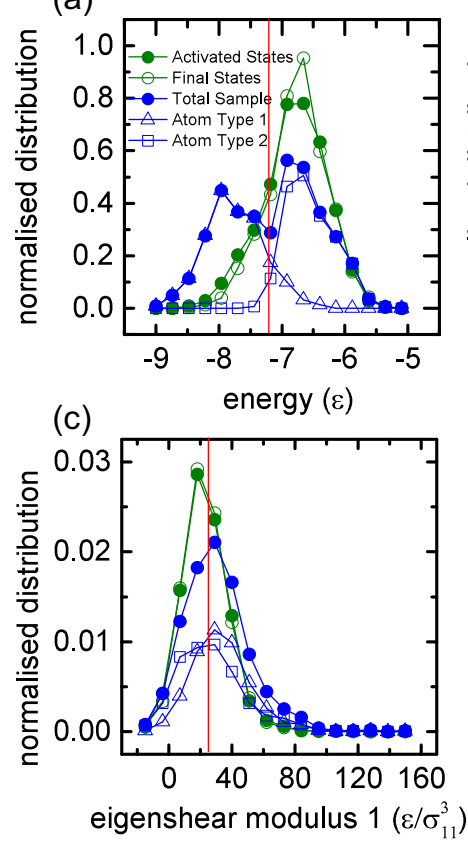

(b)

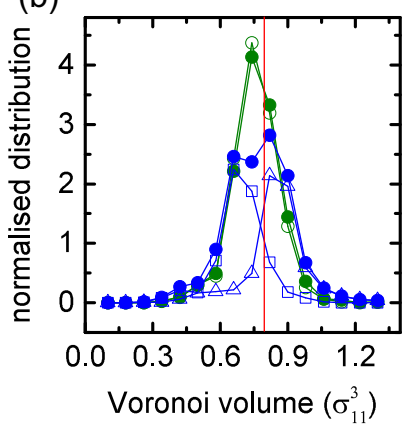

FIG. 3. (Color online) Normalized distribution of local (a) energy, (b) Voronoi volume, and (c) eigenshear modulus 1. The red vertical lines represent the corresponding mean values derived from the total sample. 
are mostly of type 2. Figure 3(b) now shows the corresponding normalized distribution for the Voronoi volume. Inspection of the unweighted curves shows that atoms of type 2 have lower volume compared to atoms of type 1 , a feature that is expected given the nature of the Wahnström parametrization (see Ref. [22]). The distinct double-peak feature is, however, absent for the LSE-weighted distributions, both the activated and final state curves showing a single-peaked structure approximately centered between the unweighted partial distributions. Closer inspection does, however, reveal a slight bias to the lower volumes of the type 2 atoms. A similar albeit weaker trend as in the Voronoi volume is seen in both the local pressure and local bulk modulus.

Figure 3(c) shows the distribution of the lowest Kelvin eigenshear modulus for the activated and final relaxed states. Again, the unweighted total and partial distributions are shown for comparison. It is noted that for each atom, the local Voigt matrix is first constructed via Eq. (7), from which the local Kelvin matrix is built and is then diagonalized (after the dilation components are projected out), to obtain five eigenshear moduli (of which the lowest eigenshear modulus is shown here). Figure 3(c) reveals that the left tail of the distribution extends into the negative moduli domain. That some atoms have a local distortion characterized by a negative modulus does not entail a local material instability, because their calculation involves only those neighboring atoms with a direct interaction and not the stabilizing effect of the more distant surrounding matrix. Such low or negative eigenshear moduli do, however, indicate the presence of local shear distortions that are soft. Inspection of the unweighted total and partial LAQ single-peak distributions reveals that atoms of type 2 are slightly biased towards regions of softer moduli. The weighted LAQ single-peak distribution follows this bias, confirming that atoms of type 2 are often involved in an LSE. No statistically significant trend is seen in the distributions of the higher Kelvin eigenshear moduli.

To gain more direct information on the type of atom involved, normalized atom type LSE-weighted distributions are generated. Figure 4(a) displays these for both the activated states and the final states. Both distributions show a maximum at an average atom-type of two, demonstrating that the most probable chemical composition of an LSE is only of type 2 atoms. This trend originates from the smaller LSEs (consisting of one to two atoms), which Fig. 2(b) demonstrates to be the most numerous. In the intermediate range of average atom type, Fig. 4(a) shows a weakly varying distribution demonstrating that on average an LSE will contain a mixture of both atom types, with a slight bias to atoms of type 2 . Inspection of such LSEs found them to consist of between three and six atoms-thus the larger LSEs consist of a mixture of both atom types. This is further demonstrated in Fig. 4(b), which shows the scatter plot of participation number with LSE-weighted atom type. Here there is a clustering of small LSEs around atom type 2, but many larger LSEs consisting of both type 1 and type 2 atoms are also evident. The lower boundary in this figure reflects the fact that for an average atom type of 1.5 at least two atoms must be involved. A closer inspection also demonstrates that LSEs with predominantly atoms of type 2 are not only more common, but they also appear to correspond to lower barrier energies. This is directly (a)
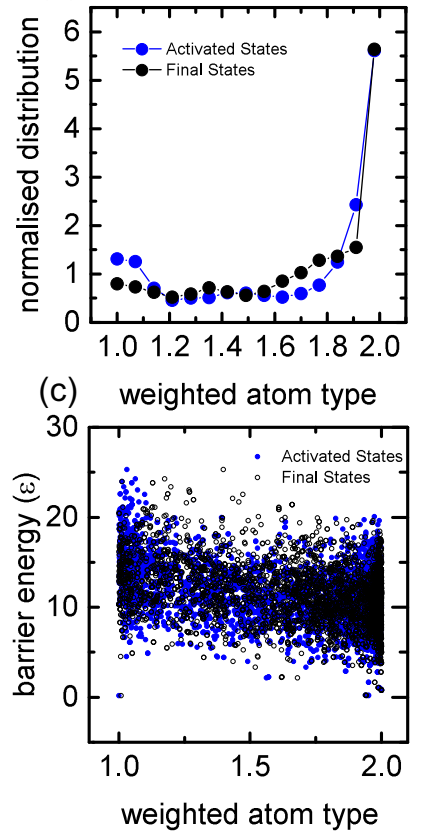

(b)

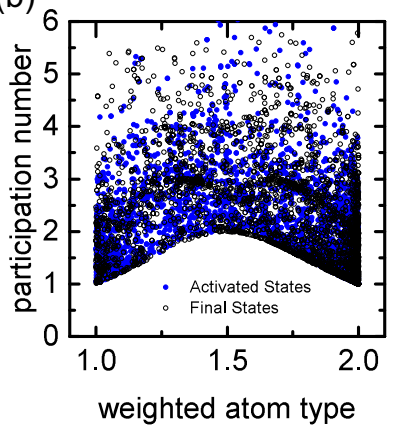

FIG. 4. (Color online) (a) Normalized distribution of LSEweighted atom types for the activated and final state configurations. (b) Scatter plot of participation number vs LSE-weighted atom type. (c) Scatter plot of barrier energy vs LSE-weighted atom type.

seen in Fig. 4(c), which is a scatter plot of LSE-weighted atom type and barrier energy. This plot shows that LSEs having a greater proportion of atoms of type 2 generally have a lower corresponding barrier energy. The scatter associated with this trend is, however, large, with both types of LSEs having a spread in barrier energy comparable to the domain of the distribution shown in Fig. 2(a).

From the perspective of thermal activation the rate of occurrence for a particular LSE is given by $v_{0} \exp \left(-E_{0} / k_{\mathrm{B}} T\right)$, where $v_{0}$ is the prefactor (rate of attempt) and $E_{0}$ is the barrier energy of the LSE. Therefore those LSEs with the lowest barrier energy will most likely occur. In the work of Koziatek et al. [21], this was demonstrated within harmonic transition state theory, where the corresponding prefactor $v$ was also calculated, showing that despite a wide range of prefactor values (spanning many orders of magnitude) those identified LSEs with lowest barrier energy generally exhibited the highest rate of occurrences. It is therefore of interest to investigate the correlation between barrier energy and the structural environment as presently defined by the LAQs of Sec. II B.

Figure 5 displays scatter plots of three of the LSE-weighted LAQs with respect to their corresponding barrier energy. Data are shown for local cohesive energy, Voronoi volume, and lowest Kelvin eigenshear modulus. While the scatter is strong for all quantities, the local cohesive energy and lowest Kelvin eigenshear modulus versus barrier energy do have non-negligible Pearson correlation coefficients equal to approximately -0.5 and 0.3 . For the case of Voronoi volume, the Pearson coefficient is approximately zero. Again, there is little statistical difference between the activated and final 
(a)

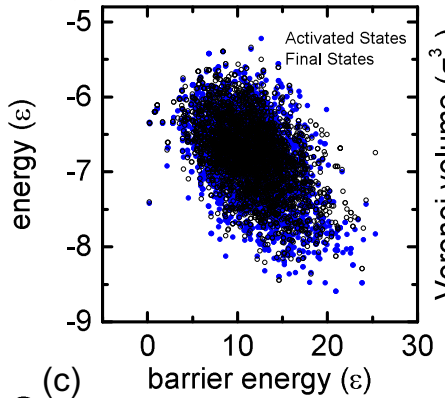

(b)

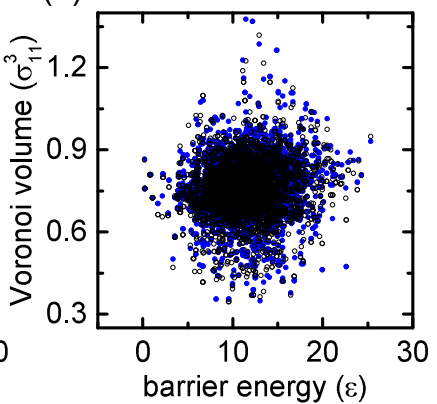

(a)

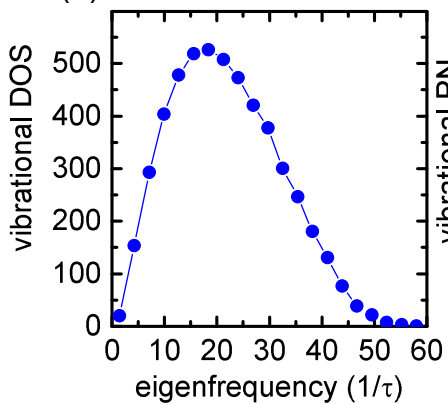

(b)

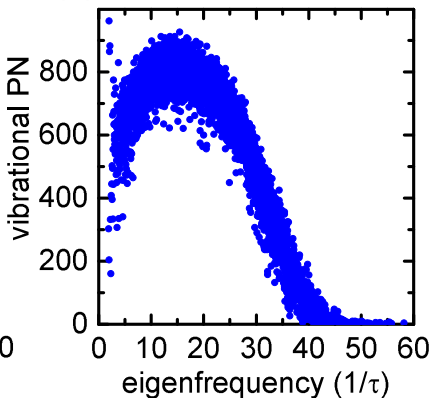

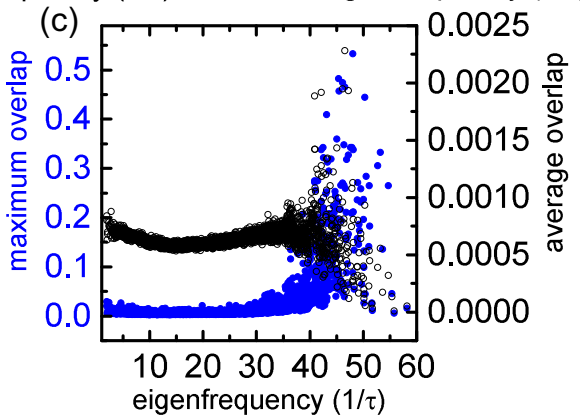

FIG. 6. (Color online) (a) Plot of vibrational density of states and (b) participation number vs eigenfrequency (in LJ units of inverse $\tau=\sqrt{m \sigma^{2} / \varepsilon}$ ). (c) Plot of maximum and average overlap per eigenfrequency.

peak, indeed, it is precisely at the transverse Ioffe-Regel frequency that the Boson peak occurs [39]. Above a certain frequency, Fig. 6(b) also reveals that the participation number begins to decrease with increasing frequency finally resulting in high-frequency eigenmodes, each involving only a few neighboring atoms. Such modes are the result of Anderson localization and occur at frequencies above a critical mobility edge value [41-44].

Previous work done by two of us has demonstrated a link between the spatial extent of the low-frequency quasilocalized modes and regions of the atomic configuration, which have a low or negative lowest eigenshear mode [22]. Moreover, high-strain molecular dynamics simulations show that at high enough temperatures, the location of irreversible structural transitions correlates strongly with regions of negative local eigenshear modulus [14]. It is therefore of interest to investigate whether or not there is a correlation between the location of an LSE and the spatial extent of the above mentioned lowfrequency quasilocalized and high-frequency localized modes. To do this, the overlap between the vibrational eigenstate and that of the LSE was determined. This was done by calculating the scalar product of the atomic weights [Eq. (2)] with the eigenvector magnitude-squared, $\left|\vec{u}_{i, n}\right|^{2}$. Figure 6(c) displays both the average overlap and the maximum overlap of all identified LSEs with each vibrational eigenvector as a function of the corresponding eigenfrequency.

The figure shows that there exists, on average, little overlap over the entire frequency range. In the low-frequency regime, the average overlap is a well-defined statistical quantity, indicating that irrespective of the nature of the quasilocalized mode, the spatial location and extension of the identified LSEs are similar for different modes. This is also reflected in the 
maximum overlap, which varies little with eigenfrequency and is also a small quantity. At higher frequencies, the situation is somewhat different in that there is much more scatter in the average value and the maximum value. This however, does not indicate any important correlation, because the small average and large maximum values more likely indicate the scenario that statistically there will be one or some LSEs that do not strongly overlap with one or some well-localized high-frequency eigenstates. This does not occur at the low-frequency quasilocalized eigenstates since these are more extended involving several tens to hundreds of atoms (see Fig. 13 of Ref. [22]). In addition, it is noted that no statistically meaningful correlation was found between the location of an LSE and that atom with the largest oscillator strength of each eigenmode. Thus the current analysis reveals little overall correlation between the spatial extent of the vibrational modes and the location of the identified LSEs.

\section{Effect of quench rates}

The ART $n$ simulations are also performed on glass samples prepared with faster quench rates (sample 0a with $\eta_{1}$, sample Ob with $\eta_{2}$, sample $0 \mathrm{c}$ with $\eta_{3}$ ) to determine if the results of Sec. III depend on the quench rate. For the more rapidly quenched systems, the peak of the barrier energy distribution is seen to shift to lower activation energies and close to the zero barrier energy limit the distribution does not reduce to zero (a result also found in the work of Rodney and Schuh [18,19]). For the case of the PN distribution [Eq. (3)], an increased quench rate correlated with a slight shift to a larger number of atoms being involved in the LSEs and many of these involve a more mixed number of atom type. These results suggest that the more rapidly the model system is quenched the more shallow the local potential minimum is, and that the corresponding LSEs are somewhat larger, involving both types (sizes) of atom. In general, however, the weak correlation with local structural features seen in Sec. III is insensitive to quench rate.

\section{ATOMIC VISUALISATION}

In this section, six identified LSEs are atomistically visualized. These examples are chosen since they represent typical features seen in all LSEs and are shown in Fig. 7. In all of the examples, the initial and final atomic positions are represented respectively by green and orange spheres, whereas the red arrows represent the displacement from the initial to activated position and the blue arrows the displacements from the activated to final position. Only those atoms are shown which are displaced by more than $0.2 \sigma$, either between the initial and activated, or the activated and final configuration. The large spheres represent atoms of type 1 and the small spheres atoms of type 2 . Generally, the visualized atoms may be classified into two groups, those central atoms that involve significant and irreversible displacement and those atoms that accommodate the activity either via reversible elastic or irreversible plastic displacement. In all figures, the first class of initial atom positions is numbered, with the dashed-corresponding-number labeling their final position. (a)

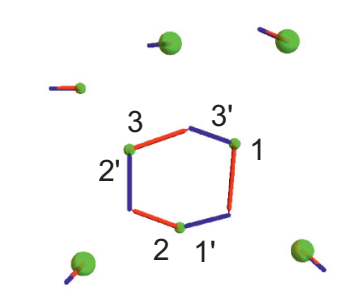

(b)

(c)

(d)
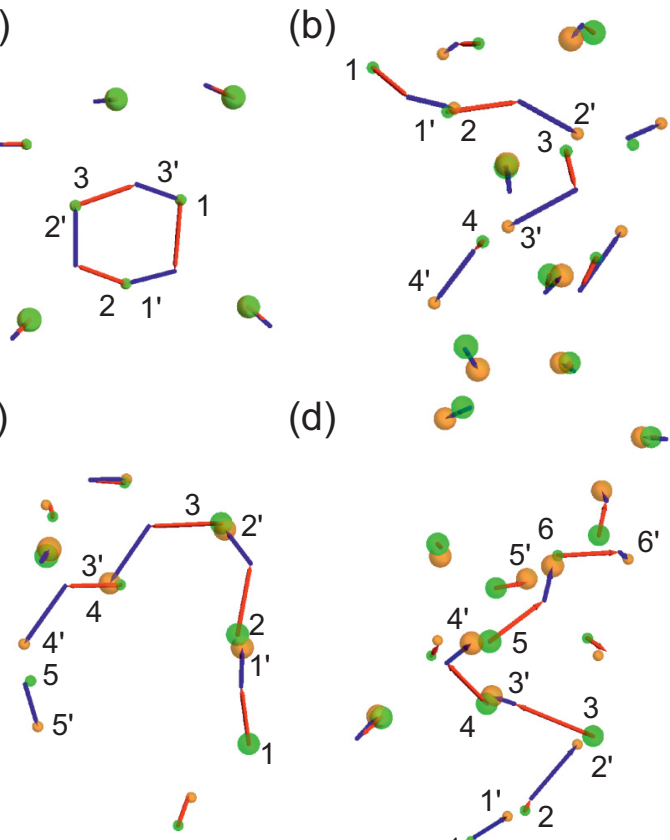

(e)

(f)

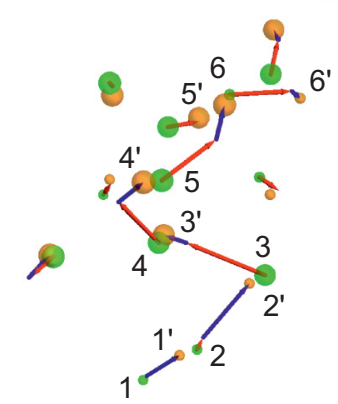

l

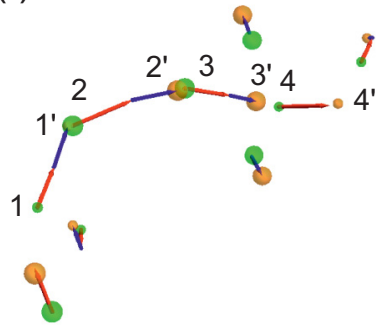

FIG. 7. (Color online) Six examples of LSEs identified by ART $n$. In each case, the initial atomic positions are visualized by green balls and the final ones by orange balls. The atomic displacements from the initial to activated and activated to final states are visualized by red and blue arrows, respectively.

Figure 7(a) represents an LSE with a barrier energy of $11.98 \epsilon$ involving eight atoms. In this LSE, the central atomic structure forms a symmetrical ringlike (or closed chainlike) structure $\left(1 \rightarrow 1^{\prime}: 2 \rightarrow 2^{\prime}: 3 \rightarrow 3^{\prime}\right)$, consisting of smaller atoms (of type 1). Surrounding this plastic inner structure, there are mainly larger atoms (of type 2), which move back and forth during the initial to activated state and then from the activated to final state transition, respectively. This is an example of an elastic accommodation mechanism around the inner ringlike plastic rearrangement. In this case, the LSE results in a final configuration identical to the initial configuration apart from a permutation of three labels. As already mentioned such LSEs are not considered in the detailed statistical analysis of Sec. II B.

Figure 7(b) represents an LSE with a barrier energy of $6.65 \epsilon$ involving 17 atoms. It shows an extended chainlike atomic motion with the sequence being specified by $(1 \rightarrow$ $1^{\prime}: 2 \rightarrow 2^{\prime}: 3 \rightarrow 3^{\prime}: 4 \rightarrow 4^{\prime}$ ). Although smaller atoms (of type 2) are involved in the formation of the chain, there is a relatively large number of large atoms (of type 1) that 
are responsible for accommodating this structural excitation. There is evidence of both elastic and plastic accommodation by the surrounding atoms, which is clearly seen by atoms moving back and forth as well as atoms moving irreversibly in the region surrounding the inner chain-like formation. From this LSE (and further confirmed in the subsequent descriptions of LSEs), it is observed that excitations, which involve a higher number of atoms in the chainlike structure also involve proportionally a higher number of atoms in the surrounding accommodation mechanism.

Figure 7(c) represents an LSE with a barrier energy of $10.35 \epsilon$ involving nine atoms. It also shows a chainlike atomic reconfiguration, now of a strongly curved extension. The sequence is specified by $\left(1 \rightarrow 1^{\prime}: 2 \rightarrow 2^{\prime}: 3 \rightarrow 3^{\prime}: 4 \rightarrow 4^{\prime}\right.$ : $5 \rightarrow 5^{\prime}$ ). Here both sized atoms are involved in the reconfiguration. This was also the case for the surrounding elastic accommodation, where both types of atoms were involved.

One of the most spatially extended LSEs identified by ARTn is shown in Fig. 7(d). This LSE has a barrier energy of $16.01 \epsilon$ and involves 17 atoms, with the reconfiguration sequence being $\left(1 \rightarrow 1^{\prime}: 2 \rightarrow 2^{\prime}: 3 \rightarrow 3^{\prime}: 4 \rightarrow 4^{\prime}: 5 \rightarrow 5^{\prime}\right.$ : $6 \rightarrow 6^{\prime}$ ). It is noted that smaller atoms (of type 2 ) are involved at both ends of the chain sequence, and that both types of atoms are involved in the elastic and plastic accommodation. Such chainlike activity (including both atom-types) is also seen in the smaller ringlike LSEs, as shown in Fig. 7(e), which has a barrier energy of $10.55 \epsilon$. Finally, Fig. 7(f) represents an LSE with a barrier energy of $8.96 \epsilon$ involving 11 atoms. This LSE forms a chain $\left(1 \rightarrow 1^{\prime}: 2 \rightarrow 2^{\prime}: 3 \rightarrow 3^{\prime}: 4 \rightarrow 4^{\prime}\right)$, which almost resembles a straight line due to its low curvature.

Upon inspection of these figures, the chainlike sequence of an LSE generally involves one atom replacing its neighbor (and so on), such that the chain or part of the chain is fully connected (with respect to the red and blue displacement arrows). In addition, one atom can move into a previously unoccupied location, with another atom doing the same with respect to another atom (and so on) forming a disconnected chain (with respect to the red and blue displacement arrows). The smaller ringlike structures of Figs. 7(a) and 7(e) fall into the first category and the extended chains fall into both categories. Very low-energy LSEs are also visualized (not shown) and these tended to involve just one atom changing its location with minor elastic and plastic accommodation in the surrounding regions. Such LSEs typically have activation energies in the range of less than $\sim 5 \varepsilon$.

\section{DISCUSSION AND CONCLUDING REMARKS}

The results of Sec. III suggest that the location of an LSE is only weakly correlated with the local structural features of those atoms involved. For the LJ system considered, the only non-negligible correlation is that the smaller atoms of type 2 are more often involved than the larger atoms of type 1, particularly when the LSEs consist of only a few atoms and are at the lower range of the barrier energy spectrum. Despite the strong scatter, this latter aspect suggests a rather intuitive scenario where type two atoms generally involve less negative bond energies. The breaking of bonds that must occur in an LSE requires less energy and therefore lower barrier energy. Indeed, this appears to be more important than local Voronoi volume.
Figure 3(b) shows that the volume LSE-weighted distribution exhibits only a central peak structure not located at volumes typical of type 2 atoms, whereas the local cohesive energy LSE distribution clearly correlates with the type 2 unweighted peak [Fig. 3(a)]. The remaining, somewhat weaker correlation with barrier energy is that a small or negative lowest local Kelvin eigenshear tends to have a low barrier energy. This is again, an intuitive result since a small or negative Kelvin eigenshear indicates a shallow potential energy minimum and therefore a smaller activation barrier. In other words, the LSEs occurring in softer regions tend to have lower activation energies.

The atomistic visualization shown in Sec. IV generally demonstrates LSEs to be a sequence of atoms that successively replace each others approximate location, with the surrounding atoms accommodating such movement through either elastic or plastic distortion. This appears to be a general result, although the spatial extension of the atomic sequence can be quite diverse, ranging from an almost linear extension to strongly curved and closed ringlike structures (for the smaller LSEs). Although those central atoms within the chain show no obvious decrease in their own local Voronoi volume, it is of interest to investigate whether nearby free volume is correlated with their existence. This is motivated by the original assumption of Spaepen in his thermally activated free-volume theory [1]. To determine the spatial location of free volume within the computer-generated sample, the simulation cell is filled with a fine regular cubic mesh of points at a spacing much smaller than the typical inter-atomic distance of $\sim \sigma$. Those mesh points that have a distance to the nearest atom greater than $R_{\max }$, and which are connected to each other, will then define the spatial extent of a region of free local volume. $R_{\max }$ cannot be too small since then the normal interstitial regions, which span the entire simulation cell, will be identified. The parameter should also not be too large since then no free volume will be identified. Such a method has been used to identify free volume in grain boundaries [52]. Using a value of $R_{\max }=0.76 \sigma$, Fig. 1 displays the identified regions as green balls. This figure shows that the computer-generated sample contains a few regions in which local free volume is above the normal background of interstitial regions.

Figure 8 now shows a histogram of $R_{\mathrm{fv}}$, the nearest distance of an LSE center-of-position (the red-colored balls in Fig. 1) to identified free volume (the green-colored balls in Fig. 1).

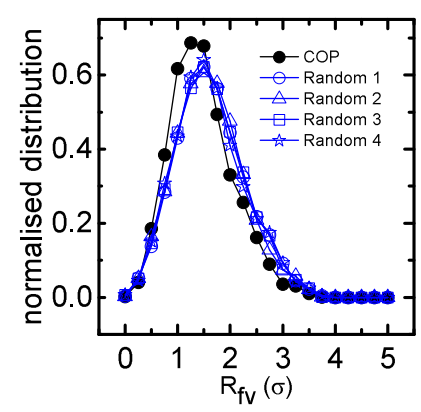

FIG. 8. (Color online) Distribution of minimum distances between the center-of-position of an LSE and free volume in the sample. Also shown for comparison are histograms of four random realizations of LSE center-of-positions derived from a uniform distribution within the simulation cell. 
Also shown are histograms of four random realizations of LSE center-of-positions derived from a uniform distribution within the simulation cell. Inspection of this figure shows a slight bias of the ART $n$ identified LSEs to be closer to free volume than that of entirely randomly located LSEs. Thus there exists some correlation between the location of an LSE and nearby free-volume.

The framework of fast $\beta$ and slower $\alpha$ structural transformations, originally developed for undercooled liquids, is now often applied to the regime of amorphous solids [45-51]. Indeed, in the work of Harmon et al. [45] multiple microscopic $\beta$ structural transformations (which are assumed to be reversible) underlay the emergence of irreversible $\alpha$ transformations in the form of a less local release of elastic energy. In more recent work [46], which attempts to explain dynamical-mechanical-spectroscopy data, the authors have postulated $\beta$ structural transformations to consist of atomic chains of smaller atoms, comparable to those encountered in the present work. On the other hand, structural transformations, which involve both small and large atoms, tend to reflect $\alpha$ transformations-a rather intuitive picture since movement of the larger atoms will tend to involve more atoms due to accommodation issues. They will therefore be inherently less local.

The usage of the terminology of $\alpha$ and $\beta$ transformations in the regime of the amorphous solid is an interesting development given that from the undercooled liquid perspective the $\alpha$ relaxations are assumed to be frozen out below the glass transition temperature [53]. How this freezing occurs and how far it extends to temperatures and affects plasticity below the glass transition has recently been considered by one of the present authors from the perspective of a thermal activation theory of deformation [49-51].

Within the above framework it is now an important question to ask, to which class of relaxation processes $(\alpha$ or $\beta$ ) the identified LSEs should belong to. Figure 9(a) displays a histogram of the change in energy between the initial and final atomic configurations found by ART $n$. In most cases, this energy is positive, with a few LSEs leading to a decrease in energy and therefore a more stable atomic configuration than the initial configuration reached by dynamical atomistic simulations. Given that only LSEs are considered that have a direct path between the initial and activated states (that is,

(a)
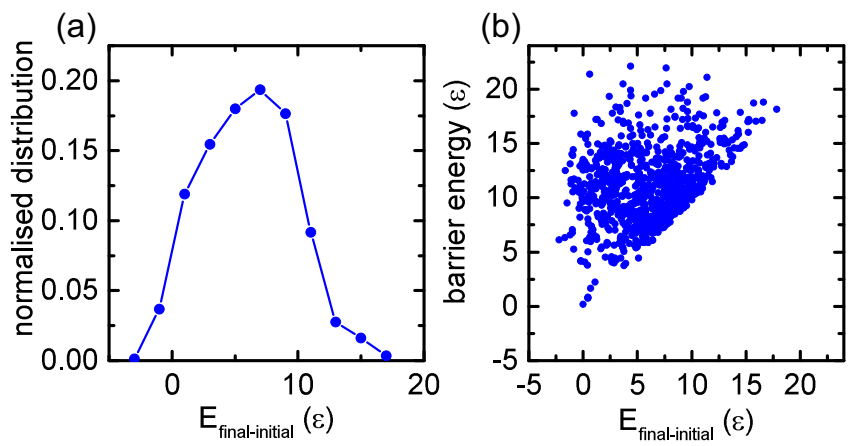

FIG. 9. (Color online) (a) Normalized distribution of the change in energy between the initial and final state configurations and (b) a scatter plot of this energy difference with the corresponding barrier energy. there exists no intermediate stable configuration), Fig. 9(a) suggests that the initial configuration is in the basin of a much larger PEL valley and therefore in the valley of the $\alpha$ landscape. From this context, the ART $n$ method appears to be probing primarily the $\beta$ PEL involving the first LSE stage that would generate the atomic configuration's journey out of its current $\alpha$ megabasin. Figure 9(b) shows the scatter plot between the change in energy between the initial and final atomic configurations with respect to the corresponding barrier energy. The plot demonstrates the obvious fact that a barrier energy cannot be less than the final-state energy for LSEs that are directly connected to the initial state. The figure also reveals that those final states that have an energy less than the initial state are separated by the full spectrum of possible activation energies, and that only very few final states have small activation energies. Generally, little correlation is seen apart from the observation that both energy scales are comparable demonstrating that, if the assumed surrounding $\alpha$ energy landscape does exist, the underlying "ripple" $\beta$ energy scale is that of the LSE energy scale. Figure 7 shows, however, that identifying LSEs as $\beta$ processes has the consequence that bonds are broken for $\beta$ processes-a result that is different from the view point that only $\alpha$ processes involve the breaking of bonds (see, for example, Ref. [54] and references therein).

Further insight into the above aspects can be gained from previous atomistic simulation work investigating structural transitions occurring in the undercooled liquid regime of a model glass [55-59]. These works all find cooperative stringlike motion involving many atoms. Detailed inspection reveals that such stringlike motion can occur either coherently, where all particles move simultaneously, or incoherently, where single particles or subgroups of particles (microstrings of the extended string structure) move sequentially in time [57]. Which behavior is more likely (either coherent or incoherent atomic motion) is believed to be temperature dependent and of entropic origin, where within the framework of randomfirst-order-transition theory a critical temperature exists above which cooperative rearrangements are characterized as fractal or "stringy," and below which they are more compact [60]. This temperature also characterizes the transition, with decreasing temperature, to an undercooled liquid regime more strongly influenced by thermal activation. Finally, in the work of Vogel et al. [58], it was found that the stringlike activity tended to facilitate irreversible structural transformations between megabasins, i.e., $\alpha$ activity, whereas incoherent activity involving only a few atoms of the string represented reversible transitions between inherent structures and therefore $\beta$ activity.

The present work therefore demonstrates that stringlike structural transformations also emerge in an entirely static regime of the PEL, where the corresponding saddle-point configuration is directly connected to the initial stable configuration via no intermediate stable configurations. This is somewhat different from the above undercooled liquid scenario, which suggests that stringlike rearrangements of atoms occur via less direct pathways, with intermediate steps each involving a subgroup of atoms belonging to the string. It is, however, noted that the dynamical stringlike excitations generally involve many more atoms than that seen in the LSEs identified with ART $n$. Together this suggests that the present LSEs are more likely to be those associated with 
the intermediate stages-the so-called micro-strings-that together lead to the cooperative atomic rearrangements seen in the undercooled liquid regime. If this is the case, the present work clearly extends the concept of the $\beta$ process to be beyond that of a simple "rattling" of an atom within its local environment without the breaking of a bond.

The above result supports the notion that the identified $\alpha$ and $\beta$ processes might not be so dissimilar-a conclusion also recently reached by $\mathrm{Ju}$ and Atzmon [61,62] via mechanical elastic-loss-modulus experiments in which the data suggest a similar underlying atomic mechanism distinguished only by STZ (or LSE) size.

In conclusion, the ARTn method has been used to identify local structural excitations (LSEs) in three-dimensional model glass samples that have been characterized in terms of their local atomic quantities (LAQ). Taking advantage of the localized displacement fields of the LSEs, the mean atomic quantity of an LSE is computed via a displacement-weighting technique and its distribution compared with total sample and atom-type-resolved distributions. It is found that only a weak correlation exists between the local atomic environment and where an LSE occurs, and its barrier energy. In particular, it is found that smaller atoms are more often involved and that those with lower barrier energy tend to occur in softer potential energy regions. The origin of this appears to lie in the weak bond energy between such atoms. In general, LSEs identified via the ARTn method occur throughout the sample, and in large number, with a slight bias to be near regions of free volume. Atomistic visualization of individual LSEs reveal them to consist of chainlike structures involving the successive replacement of one atom with that of a nearest-neighbor atom and a surrounding accommodation mechanism involving both elastic and plastic distortion.

\section{ACKNOWLEDGMENTS}

The authors thank D. Rodney and K. Samwer for helpful discussion. Support by the Swiss National Science foundation (Grant No. 200021-137871) is gratefully acknowledged.
[1] F. Spaepen, Acta Metall. 25, 407 (1977).

[2] A. Argon, Acta Metall. 27, 47 (1979).

[3] M. L. Falk and J. S. Langer, Phys. Rev. E 57, 7192 (1998).

[4] C. A. Schuh and A. C. Lund, Nat. Mater. 2, 449 (2003).

[5] C. E. Maloney and A. Lemaître, Phys. Rev. Lett. 93, 016001 (2004).

[6] A. Lemaître and C. Caroli, Phys. Rev. Lett. 103, 065501 (2009).

[7] Y. Shi and M. L. Falk, Phys. Rev. B 73, 214201 (2006).

[8] P. Guan, M. Chen, and T. Egami, Phys. Rev. Lett. 104, 205701 (2010).

[9] M. J. Demkowicz and A. S. Argon, Phys. Rev. Lett. 93, 025505 (2004).

[10] M. J. Demkowicz and A. S. Argon, Phys. Rev. B 72, 245205 (2005).

[11] M. J. Demkowicz and A. S. Argon, Phys. Rev. B 72, 245206 (2005).

[12] J. S. Langer, Phy. Rev. E 77, 021502 (2008).

[13] F. Léonforte, R. Boissière, A. Tanguy, J. P. Wittmer, and J. L. Barrat, Phys. Rev. B 72, 224206 (2005).

[14] S. G. Mayr, Phys. Rev. B 79, 060201(R) (2009).

[15] G. T. Barkema and N. Mousseau, Phys. Rev. Lett. 77, 4358 (1996).

[16] N. Mousseau and G. T. Barkema, Phys. Rev. E. 57, 2419 (1998).

[17] R. A. Olsen, G. J. Kroes, G. Henkelman, A. Arnaldsson, and H. Jónsson, J. Chem. Phys. 121, 9776 (2004).

[18] D. Rodney and C. A. Schuh, Phys. Rev. Lett. 102, 235503 (2009).

[19] D. Rodney and C. A. Schuh, Phys. Rev. B. 80, 184203 (2009).

[20] H. Kallel, N. Mousseau, and F. Schiettekatte, Phys. Rev. Lett. 105, 045503 (2010).

[21] P. Koziatek, J. L. Barrat, P. M. Derlet, and D. Rodney, Phys. Rev. B 87, 224105 (2013).

[22] P. M. Derlet and R. Maaß, and J. F. Löffler, Eur. J. Phys. B 85, 148 (2012).

[23] G. Wahnström, Phys. Rev. A 44, 3752 (1991).

[24] M. Parrinello and A. Rahman, J. App. Phys. 52, 7182 (1981).
[25] D. J. Evans and B. L. Holian, J. Chem. Phys. 83, 4069 (1985).

[26] R. J. Bell and P. Dean, Discuss. Faraday Soc. 50, 55 (1970).

[27] C. H. Rycroft, G. S. Grest, J. W. Landry, and M. Z. Bazant, Phys. Rev. E 74, 021306 (2006).

[28] J. F. Lutsko, J. Appl. Phys. 64, 1152 (1988).

[29] J. Cormier, J. M. Rickman, and T. J. Delph, J. Appl. Phys. 89, 99 (2001).

[30] W. Thomson, Phil. Trans. R. Soc. London 146, 481 (1856).

[31] G. Mavko, T. Mukerji, and J. Dvorkin, The Rock Physics Handbook (Cambridge University Press, Cambridge, UK, 2009).

[32] J. M. Carcione and F. Cavallini, Geophys. J. Int. 119, 338 (1994).

[33] H. R. Schober and B. B. Laird, Phys. Rev. B 44, 6746 (1991).

[34] V. Mazzacurati, G. Ruocco, and M. Sampoli, Europhys. Lett. 34, 681 (1996).

[35] H. R. Schober and C. Oligschleger, Phys. Rev. B 53, 11469 (1996).

[36] V. L. Gurevich, D. A. Parshin, and H. R. Schober, Phys. Rev. B 67, 094203 (2003).

[37] V. L. Gurevich, D. A. Parshin, and H. R. Schober, Phys. Rev. B 71, 014209 (2005).

[38] D. A. Parshin, H. R. Schober, and V. L. Gurevich, Phys. Rev. B 76, 064206 (2007).

[39] H. Shintani and H. Tanaka, Nat. Mat. 7, 870 (2008).

[40] G. Monaco and S. Mossa, Proc. Natl. Acad. Sci. USA 106, 16907 (2009).

[41] P. W. Anderson, Phys. Rev. 109, 1492 (1958).

[42] W. Garber, F. M. Tangerman, P. B. Allen, and J. L. Feldman, Philos. Mag. Lett. 81, 433 (2001).

[43] B. J. Huang and T.-M. Wu, Phys. Rev. E 79, 041105 (2009).

[44] P. B. Allen, K. L. Feldman, J. Fabian, and F. Wooten, Philos. Mag. B 79, 1715 (1999).

[45] J. S. Harmon, M. D. Demetriou, W. L. Johnson, and K. Samwer, Phys. Rev. Lett. 99, 135502 (2007).

[46] H. B. Yu, K. Samwer, Y. Wu, and W. H. Wang, Phys. Rev. Lett. 109, 095508 (2012). 
[47] H. B. Yu, K. Samwer, W. H. Wang, and H. Y. Bai, Nat. Commun, doi:10.1038/ncomms3204.

[48] P. M. Derlet and R. Maaß, Phys. Rev. B 84, 220201(R) (2011).

[49] P. M. Derlet and R. Maaß, Symposium NN - Structure-Property Relations in Amorphous Solids, MRS Proceedings, Vol. 1520 (Materials Research Society, Pittsburgh, 2013).

[50] P. M. Derlet and R. Maaß, Philos. Mag. B 93, 4232 (2013).

[51] P. M. Derlet and R. Maaß, arXiv:1311.3818v1 [cond-mat.soft] [Phil. Mag. B (to be published)] .

[52] H. Van Swygenhoven and P. M. Derlet, Dislocations Solids 14, 1 (2008).

[53] C. A. Angell, K. L. Ngai, G. B. McKenna, P. F. McMillan, and S. W. Martin, J. Appl. Phys. 88, 3113 (2000).

[54] D. Rodney, A. Tanguy, and D. Vandembroucq, Modelling Simul. Mater. Sci. Eng. 19, 083001 (2011).
[55] C. Donati, J. F. Douglas, W. Kob, S. J. Plimpton, P. H. Poole, and S. C. Glotzer, Phys. Rev. Lett. 80, 2338 (1998).

[56] T. B. Schrøder, S. Sastry, J. C. Dyre, and S. C. Glotzer, J. Chem. Phys. 112, 9834 (2000).

[57] Y. Gebremichael, M. Vogel, and S. C. Glotzer, J. Chem. Phys. 120, 4415 (2004).

[58] M. Vogel, B. Doliwa, A. Heuer, and S. C. Glotzer, J. Chem. Phys. 120, 4404 (2004).

[59] T. Kawasaki and A. Onuki, J. Chem. Phys. 138, 12A514 (2013).

[60] J. D. Stevenson, J. Schmalian, and P. G. Wolynes, Nat. Phys. 2, 268 (2006).

[61] J. D. Ju and M. Atzmon, Acta Mater. 74, 183 (2014).

[62] J. D. Ju and M. Atzmon, MRS Communications, available on CJOMay. doi:10.1557/mrc.2014.12. 